\title{
BACK REACTION OF STRINGS IN SELF CONSISTENT STRING COSMOLOGY
}

\author{
H.J. de $\operatorname{Vega}^{(a)}$ and N. Sánchez ${ }^{(b)}$ \\ (a) Laboratoire de Physique Théorique et Hautes Energies, Université Pierre et Marie Curie \\ (Paris VI) et Université Denis Diderot (Paris VII), Tour 16, 1er. étage, 4, Place Jussieu 75252 \\ Paris, cedex 05, France. \\ Laboratoire Associé au CNRS URA280. \\ (b) Observatoire de Paris, DEMIRM, 61, Avenue de l'Observatoire, 75014 Paris, France. \\ Laboratoire Associé au CNRS URA336, Observatoire de Paris et École Normale Supérieure.
}

(May 1994)

\begin{abstract}
We compute the string energy-momentum tensor and derive the string equation of state from exact string dynamics in cosmological spacetimes. $1+1,2+1$ and $D$-dimensional universes are treated for any expansion factor $R$. Strings obey the perfect fluid relation $p=(\gamma-1) \rho$ with three different behaviours: (i) Unstable for $R \rightarrow \infty$ with growing energy density $\rho \sim R^{2-D}$, negative pressure, and $\gamma=(D-2) /(D-1)$; (ii) Dual for $R \rightarrow 0$, with $\rho \sim R^{-D}$, positive pressure and $\gamma=D /(D-1)$ (as radiation); (iii) Stable for $R \rightarrow \infty$ with $\rho \sim R^{1-D}$, vanishing pressure and $\gamma=1$ (as cold matter). We find the back reaction effect of these strings on the spacetime and we take into account the quantum string decay through string splitting. This is achieved by considering self-consistently the strings as matter sources for the Einstein equations, as well as for the complete effective string equations. String splitting exponentially suppress the density of unstable strings for large $R$. The self-consistent solution to the Einstein equations for string dominated universes exhibits the realistic matter dominated behaviour $R \sim\left(X^{0}\right)^{2 /(D-1)}$ for large times and the radiation dominated behaviour $R \sim\left(X^{0}\right)^{2 / D}$ for early times. De Sitter universe does not emerge as solution of the effective string equations. The effective string action (whatever be the dilaton, its potential and the central charge term) is not the appropriate framework in which to address the question of string driven inflation.
\end{abstract}




\section{INTRODUCTION AND RESULTS}

Recently, several interesting progresses in the understanding of string propagation in cosmological spacetimes have been made [1]- [10]. The classical string equations of motion plus the string constraints were shown to be exactly integrable in D-dimensional de Sitter spacetime, and equivalent to a Toda-type model with a potential unbounded from below. In $2+1$ dimensions, the string dynamics in de Sitter spacetime is exactly described by the sinh-Gordon equation.

Exact string solutions were systematically found by soliton methods using the linear system associated to the problem (the so-called dressing method in soliton theory) [2, 3]. In addition, exact circular string solutions were found in terms of elliptic functions [4]. All these solutions describe one string, several strings or even an infinite number of different and independent strings. A single world-sheet simultaneously describes many different strings. This is a new feature appearing as a consequence of the interaction of the strings with the spacetime geometry. Here, interaction among the strings (like splitting and merging) is neglected, the only interaction is with the curved background. Different types of behaviour appear in the multistring solutions. For some of them the energy and proper size are bounded ('stable strings') while for many others the energy and size blow up for large radius of the universe $(R \rightarrow \infty)$ ('unstable strings'). In addition, such stable and unstable string behaviours are exhibited by the ring solutions found in ref. [6] for Friedmann-RobertsonWalker (FRW) universes and for power type inflationary backgrounds. In all these works, strings were considered as test objects propagating on the given fixed backgrounds.

In the present paper we go further in the investigation of the physical properties of the string solutions above mentioned. We compute the energy-momentum tensor of these strings and we use it to find the back reaction effect on the spacetime. That is, we investigate whether these classical strings can sustain the corresponding cosmological background. This is achieved by considering self-consistently, the strings as matter sources for the Einstein (general relativity) equations (without the dilaton field), as well as for the string effective equations (beta functions) including the dilaton, the dilaton potential and the central charge term.

In spatially homogeneous and isotropic universes,

$$
d s^{2}=\left(d X^{0}\right)^{2}-R\left(X^{0}\right)^{2} \sum_{i=1}^{D-1}\left(d X^{i}\right)^{2}
$$

the string energy-momentum tensor $T_{A}^{B}(X),(A, B=1, \ldots D)$ for our string solutions takes the fluid form, allowing us to define the string pressure $p$ through $-\delta_{i}^{k} p=T_{i}^{k}$ and the string energy density as $\rho=T_{0}^{0}$. The continuity equation $D^{A} T_{A}^{B}=0$ takes then the form

$$
\dot{\rho}+(D-1) H(p+\rho)=0
$$

where $H \equiv \frac{d \log R}{d X^{0}}$. We consider $D=1+1, D=2+1$, and generic $D$-dimensional universes.

In $1+1$ cosmological spacetimes we find the general solution of the string equations of motion and constraints for arbitrary expansion factor $R$. It consists of two families : one depends on two arbitrary functions $f_{ \pm}(\sigma \pm \tau)$ and has constant energy density $\rho$ and negative pressure $p=-\rho$. That is, a perfect fluid relation holds 


$$
p=(\gamma-1) \rho
$$

with $\gamma=0$ in $D=1+1$ dimensions. The other family of solutions depends on two arbitrary constants and describes a massless point particle (the string center of mass). This second solution has $p=\rho=u R^{-2}>0$. This is a perfect fluid type relation with $\gamma=2$. These behaviours fulfil the continuity equation (1.2) in $D=2$.

In $2+1$ dimensions and for any factor $R$, we find that circular strings exhibit three different asymptotic behaviours :

- (i) unstable behaviour for $R \rightarrow \infty$ (this corresponds to conformal time $\eta \sim \tau \rightarrow \tau_{0}$ with finite $\tau_{0}$ and proper string size $S \sim R \rightarrow \infty$ ), for which the string energy $E_{u} \sim$ $R \rightarrow \infty$ and the string pressure $p_{u} \simeq-E_{u} / 2 \rightarrow-\infty$ is negative .

- (ii) Dual to unstable behaviour for $R \rightarrow 0$. This corresponds to $\eta \sim\left(\tau-\tau_{0}\right)^{-1} \rightarrow+\infty$ for finite $\tau \rightarrow \tau_{0}, S \sim R \rightarrow 0$ (except for de Sitter spacetime where $S \rightarrow 1 / H$ ), for which the string energy $E_{d} \sim 1 / R \rightarrow \infty$ and the string pressure $p_{d} \simeq E / 2 \rightarrow+\infty$, is positive.

- (iii) Stable for $R \rightarrow \infty$, (corresponding to $\eta \rightarrow \infty, \tau \rightarrow \infty, S=$ constant ), for which the string energy is $E_{s}=$ constant and the string pressure vanishes $p_{s}=0$.

Here the indices $(u, d, s)$ stand for 'unstable', 'dual' and 'stable' respectively. The behaviours (i) and (ii) are related by the duality transformation $R \leftrightarrow 1 / R$, the case (ii) being invariant under duality. In the three cases, we find perfect fluid relations (1.3) with the values of $\gamma$ :

$$
\gamma_{u}=1 / 2 \quad, \quad \gamma_{d}=3 / 2 \quad, \quad \gamma_{s}=1
$$

For a perfect gas of strings on a comoving volume $R^{2}$, the energy density $\rho$ is proportional to $E / R^{2}$, which yields the scaling $\rho_{u}=u R^{-1}, \rho_{d}=d R^{-3}, \rho_{s}=s R^{-2}$. All densities and pressures obey the continuity equation $(1.2)$ as it must be.

The $1+1$ and $2+1$ string solutions here described exist in any spacetime dimension. Embedded in D-dimensional universes, the $1+1$ and $2+1$ solutions describe straight strings and circular strings, respectively. In D-dimensional spacetime, strings may spread in $D-1$ spatial dimensions. Their treatment has been done asymptotically in ref. [9]. We have three general asymptotic behaviours:

- (i) unstable for $R \rightarrow \infty$ with $\rho_{u}=u R^{2-D}, p_{u}=-\rho_{u} /(D-1)<0$

- (ii) Dual to unstable for $R \rightarrow 0$ with $\rho_{d}=d R^{-D}, p_{d}=\rho_{d} /(D-1)>0$.

- (iii) Stable for $R \rightarrow \infty$, with $\rho_{s}=s R^{1-D}, p_{s}=0$.

We find perfect fluid relations with the factors

$$
\gamma_{u}=\frac{D-2}{D-1} \quad, \quad \gamma_{d}=\frac{D}{D-1} \quad, \quad \gamma_{s}=1
$$

This reproduces the two dimensional and three dimensional results for $D=2$ and $D=3$, respectively. The stable regime is absent for $D=2$ due to the lack of string transverse modes there. 
The dual strings behave as radiation (massless particles) and the stable strings are similar to cold matter. The unstable strings correspond to the critical case of the so called coasting universe [11,22]. That is, classical strings provide a concrete realization of such cosmological models. The 'stable' behaviour is called 'string stretching' in the cosmic string literature 12, 13.

Strings continuously evolve from one type of behaviour to another, as is explicitly shown by our solutions [1, 使. For intermediate values of $R$, the string equation of state is clearly more complicated but a formula of the type:

$$
\rho=\left(u R+\frac{d}{R}+s\right) \frac{1}{R^{D-1}} \quad, \quad p=\frac{1}{D-1}\left(\frac{d}{R}-u R\right) \frac{1}{R^{D-1}}
$$

is qualitatively correct for all $R$ and becomes exact for $R \rightarrow 0$ and $R \rightarrow \infty$. We stress here that we obtained the string equation of state from the exact string evolution in cosmological spacetimes. Inserting the equation of state (1.6) in the Einstein-Friedmann equations of general relativity, we obtain a self-consistent solution for $R$ as a monotonically increasing function of the cosmic time $X^{0}$ [eq.(3.7)]. This string dominated universe starts at $X^{0}=$ 0 with a radiation dominated regime $R\left(X^{0}\right) \stackrel{X^{0} \rightarrow 0}{\simeq} C_{D}\left(X^{0}\right)^{\frac{2}{D}}$, then the universe expands for large $X^{0}$ as $R\left(X^{0}\right) \stackrel{X^{0}}{\simeq^{\infty}} C_{D}^{\prime}\left(X^{0}\right)^{\frac{2}{D-2}}$, which is faster than (cold) matter dominated universes (where $R \sim\left(X^{0}\right)^{\frac{2}{D-1}}$ ). For example, at $D=4, R$ grows linearly with $X^{0}$, while for matter dominated universes $R \sim\left(X^{0}\right)^{\frac{2}{3}}$.

The unstable string solutions are called in this way since their energy and invariant length grow as $R$ for large $R$. However, it must be clear that as classical string solutions they never decay. At the quantum level strings decay through splitting and we take these processes into account. Unstable strings split with a probability proportional to $R$ as $R$ increases. Long strings often split into massless strings (radiation) and a long piece [20]; that is, into a 'dual' string with energy $\sim 1 / R$ and a long string with energy $\sim R$ for large R. Now $u$ and $d$ in eqs.(1.6) become functions of the time $X^{0}$ which we find in eqs.(3.12). String decay changes drastically the large $R$ behaviour since the unstable string density $u\left(X^{0}\right)$ decreases exponentially. Taken into account string decay, our self-consistent solution $R\left(X^{0}\right)$ yields the realistic matter behaviour $R\left(X^{0}\right) \stackrel{X^{0} \rightarrow \infty}{\sim}\left(X^{0}\right)^{\frac{2}{D-1}}$. Through quantum string splitting, the unstable strings do not dominate anymore for large $R$. The stable strings (which behave as cold matter) are those surviving for $R \rightarrow \infty$. The 'dual' strings are not significantly affected by string decay and give again $R\left(X^{0}\right) \stackrel{X^{0} \rightarrow 0}{\simeq} C_{D}\left(X^{0}\right)^{\frac{2}{D}}$, the radiation type behaviour. For intermediate $R$, the three types of string behaviours (unstable, dual and stable) are present. Their cosmological implications as well as those associated with string decay deserve investigation. For a thermodynamical gas of strings the temperature $T$ as a function of $R$, scales as $1 / R$ for small $R$ (the usual radiation behaviour). Without string decay, $T$ grows as $R$ for large $R$ [eq.(3.21)]. This strange behaviour disapears when string splitting is taken into account as shown by eq.(3.22).

For the sake of completeness we analyze the effective string equations. These equations have been extensively treated in the literature [21] and they are not our central aim.

It must be noticed that there is no satisfactory derivation of inflation in the context of the effective string equations. This does not mean that string theory is not compatible with inflation, but that the effective string action approach is not enough to describe inflation. 
The effective string equations are a low energy field theory approximation to string theory containing only the massless string modes. The vacuum energy scales to start inflation are typically of the order of the Planck mass where the effective string action approximation breaks down. One must also consider the massive string modes (which are absent from the effective string action) in order to properly get the cosmological condensate yielding inflation. De Sitter inflation does not emerge as a solution of the the effective string equations.

We find that a de Sitter spacetime with Lorentzian signature self-sustained by the strings necessarily requires a constant imaginary part $\pm i \pi$ in the dilaton field $\Psi$, telling us that the gravitational constant $G \sim e^{\Psi}<0$, here describes antigravity [see eqs.(1.21)]. In the

euclidean signature case $(+++\ldots+)$, we find a constant curvature solution self-sustained by the strings with a real dilaton and $G \sim e^{\Psi}>0$, but of anti-de Sitter type. Both self-consistent solutions, de Sitter and anti-de Sitter are mapped one into another by the transformation (4.22).

The outline of this paper is as follows. Section II deals with the string propagation and the string energy-momentum tensor in cosmological spacetimes. (In sections II.A, II.B and II.C we treat the $1+1,2+1$ and $D$-dimensional cases respectively and derive the corresponding string equations of state). In section III we treat self-consistent string cosmology including the string equations of state and the quantum string decay. (Section III.A deals with general relativity, III.B with the quantum string decay and III.C with the effective (beta functions) string equations).

\section{STRING PROPAGATION IN COSMOLOGICAL SPACETIMES}

We obtain in this section physical string properties from the exact string solutions in cosmological spacetimes.

We consider strings in spatially homogeneous and isotropic universes with metric

$$
d s^{2}=\left(d X^{0}\right)^{2}-R\left(X^{0}\right)^{2} \sum_{i=1}^{D-1}\left(d X^{i}\right)^{2}
$$

where $X^{0}$ is the cosmic time. In terms of the conformal time

$$
\eta=\int^{X^{0}} \frac{d X^{0}}{R\left(X^{0}\right)}
$$

the metric (2.1) takes the form

$$
d s^{2}=R(\eta)^{2}\left[(d \eta)^{2}-\sum_{i=1}^{D-1}\left(d X^{i}\right)^{2}\right]
$$

The classical string equations of motion can be written here as

$$
\begin{gathered}
\partial^{2} X^{0}-R\left(X^{0}\right) \frac{\mathrm{d} R}{\mathrm{~d} X^{0}} \quad \sum_{i=1}^{D-1}\left(\partial_{\mu} X^{i}\right)^{2}=0, \\
\partial_{\mu}\left[R^{2} \partial^{\mu} X^{i}\right]=0, \quad 1 \leq i \leq D-1,
\end{gathered}
$$

and the constraints are 


$$
T_{ \pm \pm}=\left(\partial_{ \pm} X^{0}\right)^{2}-R\left(X^{0}\right)^{2}\left(\partial_{ \pm} X^{i}\right)^{2}=0
$$

The spacetime string energy-momentum tensor is given by:

$$
\sqrt{-G} T^{A B}(X)=\frac{1}{2 \pi \alpha^{\prime}} \int d \sigma d \tau\left(\dot{X}^{A} \dot{X}^{B}-X^{A} X^{B}\right) \delta^{(D)}(X-X(\sigma, \tau))
$$

where dot and prime stands for $\partial / \partial \tau$ and $\partial / \partial \sigma$, respectively.

As we will see below, $T^{A B}(X)$ takes the fluid form for string solutions allowing us to define the string pressure $p$ and energy density $\rho$ :

$$
T_{A}^{B}=\left(\begin{array}{rrrr}
\rho & 0 & \cdots & 0 \\
0 & -p & \cdots & 0 \\
\cdots & \cdots & \cdots & 0 \\
0 & 0 & \cdots & -p
\end{array}\right)
$$

Notice that the continuity equation

$$
D^{A} T_{A}^{B}=0
$$

takes here the form

$$
\dot{\rho}+(D-1) H(p+\rho)=0
$$

where $H \equiv \frac{1}{R} \frac{d R}{d X^{0}}$.

For an equation of state of the type of a perfect fluid, that is

$$
p=(\gamma-1) \rho \quad, \quad \gamma=\text { constant }
$$

eqs.(2.8) and (2.9) can be easily integrated with the result

$$
\rho=\rho_{0} R^{\gamma(1-D)} .
$$

For $\gamma=1$ this corresponds to cold matter $(p=0)$ and for $\gamma=\frac{D}{D-1}$ this describes radiation with $p=\frac{\rho}{D-1}$.

\section{A. 1+1 Dimensional Universes}

Let us start by considering strings in this simpler case. Here $D=2$ and the metric (2.1) takes the form

$$
d s^{2}=\left(d X^{0}\right)^{2}-R\left(X^{0}\right)^{2}(d X)^{2}
$$

It is convenient to start by solving the constraints (2.5)

$$
\left(\partial_{ \pm} X^{0}\right)^{2}=R\left(X^{0}\right)^{2}\left(\partial_{ \pm} X\right)^{2}
$$

They reduce to

$$
\partial_{ \pm} X^{0}=\epsilon_{ \pm} \quad R\left(X^{0}\right) \partial_{ \pm} X
$$


where $\epsilon_{ \pm}^{2}=1$. Using the conformal time (2.2), eq.(2.12) yields

$$
\partial_{ \pm}\left(\eta-\epsilon_{ \pm} X\right)=0 .
$$

We find a first family of solutions choosing $\epsilon_{ \pm}= \pm 1$. Then

$$
\eta+X=\phi(\sigma+\tau), \quad \eta-X=\chi(\sigma-\tau)
$$

Where $\phi$ and $\chi$ are arbitrary functions of one variable. It is easy now to check that eq.(2.13) fulfills the string equations of motion (2.4).

The solution (2.13) is analyzed in detail for de Sitter spacetime $\left(R\left(X^{0}\right)=e^{H X^{0}}\right)$ in ref. [1] where the global topology of the space is taken into account.

Since one can always perform conformal transformations

$$
\sigma+\tau \rightarrow f(\sigma+\tau) \quad, \quad \sigma-\tau \rightarrow g(\sigma-\tau),
$$

with arbitrary functions $f$ and $g$, the solution (2.13) has no degrees of freedom other than topological ones.

Let us compute the energy momentum tensor for the string solution (2.13). We find from eqs.(2.6) and (2.13),

$$
\begin{aligned}
\sqrt{-G} T^{00}(X) & =\frac{1}{2 \pi \alpha^{\prime}} \int d \sigma d \tau\left(\dot{\eta}^{2}-\eta^{\prime 2}\right) \delta(\eta-\eta(\sigma, \tau)) \delta(X-X(\sigma, \tau)) \\
& =\frac{1}{2 \pi \alpha^{\prime}} \frac{\dot{\eta}^{2}-\eta^{\prime 2}}{J}
\end{aligned}
$$

where $J=\frac{\partial(X, \eta)}{\partial(\sigma, \tau)}$ is the jacobian. From eq. (2.13) we find $J=-\chi^{\prime} \phi^{\prime}$ and $\dot{\eta}^{2}-\eta^{\prime 2}=-\chi^{\prime} \phi^{\prime}$. Then,

$$
\sqrt{-G} T^{00}(X)=\frac{1}{2 \pi \alpha^{\prime}} .
$$

We analogously find $\dot{X}^{2}-X^{\prime 2}=-\chi^{\prime} \phi^{\prime}$. Then

$$
\sqrt{-G} T^{11}(X)=-\frac{1}{2 \pi \alpha^{\prime}} \quad, \quad T^{01}(X)=0 .
$$

That implies,

$$
\rho=\frac{1}{2 \pi \alpha^{\prime}}, \quad p=-\frac{1}{2 \pi \alpha^{\prime}}, \quad p+\rho=0 .
$$

We find a constant energy density and a constant negative pressure. They exactly fulfill the continuity equation (2.8). These results hold for arbitrary cosmological spacetimes in $1+1$ dimensions. That is, for arbitrary factors $R\left(X^{0}\right)$. In particular they are valid for strings winded $n$-times around the de Sitter universe [1].

A second family of string solutions follows from eq.(2.11) by choosing

$$
\eta= \pm X+C_{ \pm}
$$

where $C_{ \pm}$is a constant. Then, the string equations of motion (2.4) become 


$$
\partial_{\mu}\left[R(\eta)^{2} \partial^{\mu} \eta\right]=0
$$

Using eq.(2.16), we find that the energy-momentum tensor (2.6) is traceless for this string solution:

$$
T^{00}=T^{11} \quad, \quad \text { that is } p=\rho
$$

Let us call

$$
V(\eta)=\int^{\eta} R^{2}(x) d x
$$

Then, eq.(2.17) implies that

$$
\left(\frac{\partial^{2}}{\partial \tau^{2}}-\frac{\partial^{2}}{\partial \sigma^{2}}\right) V(\eta)=0 .
$$

The general solution $\eta=\eta(\sigma, \tau)$ is implicitly defined by:

$$
\int^{\eta} R^{2}(x) d x=A(\sigma-\tau)+B(\sigma+\tau) .
$$

where $A(x)$ and $B(x)$ are arbitrary functions. Upon a conformal transformation, without loss of generality we can set

$$
A(\sigma-\tau)+B(\sigma+\tau) \Rightarrow \tau .
$$

Hence, $\eta$ is in general a function solely of $\tau$ with

$$
\tau=\int^{\eta} R^{2}(x) d x \text { or } \frac{d \eta}{d \tau}=\frac{1}{R^{2}(\eta)} .
$$

The energy-momentum tensor (2.6) takes for this solution the form:

$$
T_{A}^{B}=\frac{1}{\alpha^{\prime} R^{2}} \delta\left(\eta \mp X-C_{ \pm}\right)\left(\begin{array}{rr}
1 & 0 \\
0 & -1
\end{array}\right)
$$

The $\delta\left(\eta \mp X-C_{ \pm}\right)$characterizes a localized object propagating on the characteristics at the speed of light. This solution describes a massless point particle since it has been possible to gauge out the $\sigma$ dependence .

In summary, the two-dimensional string solutions in cosmological spacetimes generically obey perfect fluid equations of state with either

$$
p=-\rho(\gamma=0) \quad \text { or } \quad p=+\rho \quad(\gamma=2)
$$

The respectives energy densities being

$$
\rho=\rho_{u} \quad\left(\rho_{u}=\text { constant }\right) \text { for } \gamma=0 \quad \text { or } \quad \rho=\frac{u}{R^{2}} \quad(u=\text { constant }) \text { for } \gamma=2 .
$$

These behaviors fulfill the continuity equation (2.8) for $D=2$. 


\section{B. 2+1 Dimensional Universes}

In $2+1$ dimensions, a large class of exact solutions describing one string and multistrings has been found in de Sitter universe [2] - [5]. For power-like expansion factors $R(\eta)^{2}=$ $A \eta^{k},(k \neq-2)$ only ring solutions are known [6]. $(k=-2$ corresponds to de Sitter spacetime). Ring solutions correspond to the Ansatz [6]:

$$
\begin{aligned}
& X^{0}=X^{0}(\tau) \\
& X^{1}=f(\tau) \cos \sigma \\
& X^{2}=f(\tau) \sin \sigma
\end{aligned}
$$

The total energy of one string is then given by (recall $G^{00}=1$ )

$$
E\left(X^{0}\right)=\int d^{D-1} X \sqrt{-G} T^{00}(X)=\frac{1}{\alpha^{\prime}} \frac{d X^{0}}{d \tau}
$$

More generally, the energy-momentum tensor integrated on a volume that completely encloses the string, takes the form [10]

$$
\begin{aligned}
\Theta^{A B}(X) & =\frac{1}{2 \pi \alpha^{\prime}} \int d \sigma d \tau\left(\dot{X}^{A} \dot{X}^{B}-X^{A} X^{B}\right) \delta\left(X^{0}-X^{0}(\tau)\right) \\
& =\frac{1}{2 \pi \alpha^{\prime}\left|\dot{X}^{0}(\tau)\right|} \int_{0}^{2 \pi} d \sigma\left[\dot{X}^{A} \dot{X}^{B}-X^{\prime A} X^{\prime B}\right]_{\tau=\tau\left(X^{0}\right)}
\end{aligned}
$$

For multistring solutions, one must sum over the different roots $\tau_{i}$ of the equation $X^{0}=$ $X^{0}(\tau)$, for a given $X^{0}$.

We find for the ring ansatz eq.(2.22):

$$
\begin{aligned}
& \Theta^{00}(X)=E\left(X^{0}\right) \\
& \Theta^{11}(X)=\Theta^{22}(X)=\frac{1}{2 \alpha^{\prime}\left|\dot{X}^{0}(\tau)\right|}\left[\dot{f}^{2}-f^{2}\right] \\
& \Theta^{01}(X)=\Theta^{02}(X)=\Theta^{12}(X)=0
\end{aligned}
$$

That is,

$$
\Theta_{A}^{B}=\left(\begin{array}{rrr}
E & 0 & 0 \\
0 & -P & 0 \\
0 & 0 & -P
\end{array}\right)
$$

where,

$$
E=\frac{1}{\alpha^{\prime}} \dot{X}^{0}(\tau) \quad, \quad P=\frac{R(\tau)^{2}}{2 \alpha^{\prime}\left|\dot{X}^{0}(\tau)\right|}\left[\dot{f}^{2}-f^{2}\right]
$$

Let us first consider ring strings in de Sitter universe [2] - [4]. There are three different asymptotic behaviors: stable, unstable and its dual. The unstable regime appears for $\eta \simeq$ $\frac{\tau}{H} \rightarrow 0$. From eqs. 2.22) and ref. [2], we find 


$$
\begin{aligned}
& E(\tau) \stackrel{\tau \rightarrow 0^{-}}{=} \frac{1}{\alpha^{\prime} H}\left(\frac{1}{|\tau|}+1\right) \simeq \frac{1}{\alpha^{\prime} H}(R(\tau)+1) \rightarrow+\infty \\
& P(\tau) \stackrel{\tau \rightarrow 0^{-}}{=}-\frac{1}{2 \alpha^{\prime} H|\tau|} \simeq-\frac{R(\tau)}{2 \alpha^{\prime} H} \rightarrow-\infty
\end{aligned}
$$

(Here $H$ stands for the Hubble constant). The invariant string size grows as $R / H$ in this unstable regime. Notice that the pressure is negative for the unstable strings and proportional to the expansion factor $R$. In this regime we also see that

$$
P \stackrel{R \rightarrow \infty}{=}-\frac{E}{2}+\frac{1}{2 \alpha^{\prime} H}+O(1 / R) \rightarrow-\infty
$$

with $E=\frac{1}{\alpha^{\prime} H}(R(\tau)+1)$. The regime dual to the unstable regime appears when the conformal time $\eta$ tends to infinity. For the solution $q_{-}(\sigma, \tau)$ of ref. [2] in de Sitter universe, $\eta$ diverges for finite $\tau \rightarrow \tau_{0}$ as

$$
\eta \stackrel{\tau \rightarrow \tau_{0}}{=} \frac{6 e^{-\tau_{0}}}{\tau-\tau_{0}}+O(1) \rightarrow+\infty
$$

Here

$$
\tanh \frac{\tau_{0}}{\sqrt{2}}=\frac{1}{\sqrt{2}} \quad, \quad \tau_{0}=1.246450 \ldots
$$

Then,

$$
\begin{aligned}
& R(\tau) \stackrel{\tau \rightarrow \tau_{0}}{=} \frac{e^{\tau_{0}}}{6}\left(\tau-\tau_{0}\right) \rightarrow 0^{+} \\
& E(\tau) \stackrel{\tau \rightarrow \tau_{0}}{=} \frac{1}{\alpha^{\prime} H\left(\tau-\tau_{0}\right)}=\frac{0.5796 \ldots}{\alpha^{\prime} H R} \rightarrow+\infty \\
& P(\tau) \stackrel{\tau \rightarrow \tau_{0}}{=} \frac{1}{2 \alpha^{\prime} H\left(\tau-\tau_{0}\right)}=E / 2 \rightarrow+\infty
\end{aligned}
$$

We call dual to this regime since it appears related to the unstable regime (2.23) through the exchange $R \leftrightarrow 1 / R$. The invariant string size tends to $\frac{1}{H}$ in this regime.

In the stable regime, $\tau \rightarrow \infty$, (and the cosmic time $X^{0} \simeq \frac{\tau}{H} \rightarrow \infty$ ), from eq.([2.22) and ref. [2], we find

$$
E(\tau) \stackrel{\tau \rightarrow \infty}{=} \frac{1}{\alpha^{\prime} H} \quad, \quad P(\tau) \stackrel{\tau \rightarrow \infty}{=} \frac{1+\sqrt{2}}{2 H} e^{-\tau \sqrt{2}} \rightarrow 0
$$

For stable strings in de Sitter universe, the pressure is positive and vanishes asymptotically, and the invariant string size tends to $\frac{1}{\sqrt{2} H}$.

The solution $q_{-}(\sigma, \tau)$ in ref. [2] describes two ring strings: A stable string for $q_{0} \rightarrow$ $+\infty(\tau \rightarrow \infty)$ ( $q_{0}$ being the hyperboloid time-like coordinate) and a unstable one for $q_{0} \rightarrow$ $-\infty\left(\tau \rightarrow \tau_{0}\right)$. The pressure $P$ depends on $\tau$; it is negative for $q_{0}<l$ and positive for $q_{0}>l$, where $l=-1.385145 \ldots$

It can be noticed that the behaviour eq.(2.23) for the energy can be interpreted as an unstable piece $R /\left(\alpha^{\prime} H\right)$ plus a stable one $1 /\left(\alpha^{\prime} H\right)$. The constant term $1 /\left(\alpha^{\prime} H\right)$ is precisely the energy for the stable solution eq.(2.24). 
Let us now study the energy and pressure for the ring string solutions in FRW and power-type inflationary universes considered in [6]. In terms of the conformal time $\eta$, we have as expansion factor $R^{2}(\eta)=A \eta^{k}$.

Near $\eta=0$, two types of behavior were found [6]. The first one is a linear behavior

$$
\begin{gathered}
\eta \stackrel{\tau \rightarrow \tau_{0}}{=} \tau-\tau_{0}+O\left(\tau-\tau_{0}\right)^{2} \\
f(\tau) \stackrel{\tau \rightarrow \tau_{0}}{=} 1-\frac{\left(\tau-\tau_{0}\right)^{2}}{2(k+1)} \text { for } k>0 \text { and } k<-1 \\
\text { and } f(\tau) \stackrel{\tau \rightarrow \tau_{0}}{=} 1+c\left(\tau-\tau_{0}\right)^{1-k} \text { for }-1<k<0 \text {. }
\end{gathered}
$$

Eqs.(2.25) describe a collapsing (expanding) string for $k>0(k<0)$ with invariant size $\simeq\left(\tau-\tau_{0}\right)^{k / 2}$. That is, in standard FRW universes $(k>0)$, the string size goes to zero for $\eta \simeq\left(\tau-\tau_{0}\right) \rightarrow 0$ as the universe radius $R \simeq\left(\tau-\tau_{0}\right)^{k / 2} \rightarrow 0$. In inflationary universes $(k<0)$, the string size grows indefinitely for $\eta \simeq\left(\tau-\tau_{0}\right) \rightarrow 0$ as the universe radius $R \simeq\left(\tau-\tau_{0}\right)^{k / 2} \rightarrow+\infty$. The growing of the proper string size for $\eta \simeq \tau-\tau_{0} \rightarrow 0$ is a typical feature of string instability.

Using eqs.(2.22) and (2.25), the energy and pressure take the form,

$$
\begin{aligned}
& E(\tau) \stackrel{\tau \rightarrow \tau_{0}}{=} \frac{\sqrt{A}}{\alpha^{\prime}}\left(\tau-\tau_{0}\right)^{k / 2}=R / \alpha^{\prime}, \\
& P(\tau) \stackrel{\tau \rightarrow \tau_{0}}{=}-\frac{\sqrt{A}}{2 \alpha^{\prime}}\left(\tau-\tau_{0}\right)^{k / 2}=-R /\left(2 \alpha^{\prime}\right) .
\end{aligned}
$$

That yields

$$
P \stackrel{\tau \rightarrow \tau_{0}}{=}-E / 2
$$

Notice that eq.(2.27) holds both for $R \rightarrow \infty$ in power-like inflationary universes $(k<0)$ and for $R \rightarrow 0$ in standard FRW universes $(k>0)$.

Eq.(2.27) is also valid in de Sitter universe for unstable strings [eq.(2.23)]. In all these cases strings exhibit negative pressure.

The second behavior present near $\eta=0$ is [6]

$$
\eta \stackrel{\tau \rightarrow \tau_{0}}{=}\left(\tau-\tau_{0}\right)^{1 /(k+1)} \quad, \quad f(\tau) \stackrel{\tau \rightarrow \tau_{0}}{=} f_{0} \pm\left(\tau-\tau_{0}\right)^{1 /(k+1)}
$$

where $f_{0}$ must be set equal to zero for $-1<k<0$. The invariant string size behaves for $\tau \rightarrow \tau_{0}$ as,

$$
\begin{array}{ll}
S(\tau) \stackrel{\tau \rightarrow \tau_{0}}{=} \sqrt{A}\left(\tau-\tau_{0}\right)^{\frac{k+2}{2(k+1)}} & \text { for } k<0 \\
S(\tau) \stackrel{\tau \rightarrow \tau_{0}}{=} \sqrt{A}\left(\tau-\tau_{0}\right)^{\frac{k}{2(k+1)}} & \text { for } k>0
\end{array}
$$

This solution describes a string that collapses for $k>0$ and for $k<-2$, that is, both for standard FRW and inflationary cosmologies. Here, the expansion factor tends to zero as

$$
R(\tau) \stackrel{\tau \rightarrow \tau_{0}}{=} \sqrt{A}\left(\tau-\tau_{0}\right)^{\frac{k}{2(k+1)}} \rightarrow 0
$$

when $k>0$ and when $k<-1$. 
That is, in this case, for small radius $R \rightarrow 0$ of both standard FRW and inflationary cosmologies, strings collapse.

From eq. 2.22) we find that

$$
P=\frac{A}{2(k+1) \alpha^{\prime} R} \rightarrow+\infty \quad, \quad E=\frac{A}{(k+1) \alpha^{\prime} R} \rightarrow+\infty
$$

That is,

$$
P \stackrel{\tau \rightarrow \tau_{0}}{=} E / 2
$$

Notice that the pressure is here positive. This second behaviour is related by duality $(R \leftrightarrow 1 / R)$ to the first behaviour described by eqs.(2.26)-(2.27).

For large $\tau$, the ring strings exhibit a stable behaviour [6],

$$
\begin{aligned}
\eta(\tau) & \stackrel{\tau \rightarrow+\infty}{=} \tau^{2 /(k+2)}, \\
X^{0}(\tau) & \stackrel{\tau \rightarrow+\infty}{=} \frac{2 \sqrt{A}}{k+2} \tau, \\
f(\tau) & \stackrel{\tau \rightarrow+\infty}{=} \frac{2}{k+2} \tau^{-k /(k+2)} \cos (\tau+\varphi),
\end{aligned}
$$

where $\varphi$ is a constant phase and the oscillation amplitude has been normalized. For large $\tau$ , the energy and pressure of the solution (2.30) behave as

$$
\begin{aligned}
& E(\tau) \stackrel{\tau \rightarrow+\infty}{=} \frac{2 \sqrt{A}}{\alpha^{\prime}(k+2)}=\text { constant }, \\
& P(\tau) \stackrel{\tau \rightarrow+\infty}{=}-\frac{\sqrt{A}}{\alpha^{\prime}(k+2)} \cos (2 \tau+2 \varphi) \rightarrow 0
\end{aligned}
$$

This is the analog of the stable behaviour (2.24) in de Sitter universe for $\tau \rightarrow \infty$. Notice that eqs. 2.31) hold both for standard FRW $(k>0)$ and inflationary spacetimes $(k<0)$.

For power-like inflationary universes with $k<-1$ a special exact ring solution exist [6] with

$$
\eta=C \exp \frac{\tau}{\sqrt{-k-1}} \quad, \quad f(\tau)=\frac{C}{\sqrt{-k}} \exp \frac{\tau}{\sqrt{-k-1}}
$$

where $C$ is an arbitrary constant. For $k=-2$ (de Sitter universe) this is the solution $q^{(o)}(\sigma, \tau)$ in ref. [2] which has constant string size.

For this solution we find

$$
\begin{aligned}
& E=\frac{1}{\alpha^{\prime}} \sqrt{\frac{A}{-k-1}} \exp \left[\frac{\tau(k+2)}{2 \sqrt{-k-1}}\right]=K R^{1+2 / k} \\
& P=-\left(\frac{1}{2}+\frac{1}{k}\right) E
\end{aligned}
$$

where $K$ is a constant. 
This is a fluid-like equation of state with $\gamma=\frac{1}{2}-\frac{1}{k}$. Notice that $\frac{1}{2}<\gamma<\frac{3}{2}$. For this solution, the energy grows with $R$ as $R$ to a power $1+2 / k$ where, since $k<-1$,

$$
-1<1+\frac{2}{k}<1
$$

( $E$ and $P$ of this solution are constants in de Sitter spacetime $[k=-2]$ ). This means that these special strings are subdominant both for $R \rightarrow \infty$ and for $R \rightarrow 0$ where the unstable strings $(E \simeq R)$ and their dual $\left(E \simeq R^{-1}\right)$ dominate respectively.

In summary, three asymptotic behaviors are exhibited by ring solutions.

i) Unstable for $R \rightarrow \infty: E=$ (constant) $R \rightarrow+\infty, P=-E / 2 \rightarrow-\infty, S \simeq R \rightarrow \infty$.

ii) Dual to unstable for $R \rightarrow 0: E=$ (constant) $R^{-1} \rightarrow+\infty, P=+E / 2 \rightarrow+\infty$, $S \simeq R \rightarrow 0$ (except for de Sitter where $S \rightarrow \frac{1}{H}$ ).

iii) Stable for $R \rightarrow \infty: E=$ constant , $P=0, S=$ constant .

In addition we have the special behavior (2.33) for $k<-1$. Notice that the three behaviors i)-iii) appear for all expansion factors $R(\eta)$. The behaviours i) and ii) are related by the duality transformation $R \leftrightarrow 1 / R$, the case iii) being invariant under duality . In the three cases we find perfect fluid equations [see eq.(2.9)] with different $\gamma$ :

$$
\gamma_{u}=1 / 2, \quad \gamma_{d}=3 / 2, \gamma_{s}=1
$$

where the indices $u, d$ and $s$ stand for 'unstable', 'dual' and 'stable', respectively. Assuming a perfect gas of strings on a volume $R^{2}$, the energy density $\rho$ will be proportional to $E / R^{2}$. This yields the following scaling with the expansion factor using the energies from i)-iii):

$$
\rho_{u}=\text { constant } R^{-1}, \rho_{d}=\text { constant } R^{-3}, \rho_{s}=\text { constant } R^{-2} .
$$

All three densities and pressures obey the continuity equation (2.8), as it must be.

The factor $1 / 2$ in the relation between $P$ and $E$ for the cases i) and ii) is purely geometric. Notice that this factor was one in $1+1$ dimensions [eq.(2.19)].

\section{D-Dimensional Universes}

The solutions investigated in secs. II.A and II.B can exist for any dimensionality of the spacetime. Embedded in D-dimensional universes, the $(1+1)$ solutions of section II.A describe straight strings, the $(2+1)$ solutions of section II.B are circular rings. In Ddimensional spacetime, one expects string solutions spread in $D-1$ spatial dimensions. Their treatment has been done asymptotically in ref. [9]. One finds for $\eta \simeq \tau-\tau_{0} \rightarrow 0$, $R \rightarrow \infty$,

$$
P_{u}=-\frac{1}{D-1} \rho_{u} \quad, \quad \gamma_{u}=\frac{D-2}{D-1} \quad \text { for unstable strings }
$$

This relation coincides with eq.(2.15) for $D=2$ and with eqs.(2.23) and (2.27) for $D=3$. 
The energy density scales with $R$ as

$$
\rho_{u}=u R^{2-D} \quad, \quad R \rightarrow \infty
$$

(where $u$ is a constant) in accordance with eq.(2.20) for $D=2$ and with eq.(2.33) for $D=3$.

For the dual regime, $R \rightarrow 0$, we have:

$$
P_{d}=+\frac{1}{D-1} \rho_{d} \quad, \quad \gamma_{d}=\frac{D}{D-1} \text { with } \rho_{d}=d R^{-D} \quad, \quad R \rightarrow 0,
$$

(where $d$ is a constant). Eq.(2.36) reduces to eq.(2.18) for $D=2$ and to eqs.(2.24) and (2.29) for $D=3$. In this dual regime strings have the same equation of state as massless radiation.

Finally, for the stable regime we have

$$
P_{s}=0, \gamma_{s}=1 \text { with } \rho_{s}=s R^{1-D}
$$

(where $s$ is a constant). This regime is absent in $D=2$ and appears for $D=3$ solutions in eqs.(2.24) and (2.31). The lack of string transverse modes in $D=2$ explains the absence of the stable regime there. The equation of state for stable strings coincides with the one for cold matter.

In conclusion, an ideal gas of classical strings in cosmological universes exhibit three different thermodynamical behaviours, all of perfect fluid type:

1) Unstable strings : negative pressure gas with $\gamma_{u}=\frac{D-2}{D-1}$

2) Dual behaviour : positive pressure gas similar to radiation, $\gamma_{d}=\frac{D}{D-1}$

3) Stable strings : positive pressure gas similar to cold matter, $\gamma_{s}=1$.

The unstable string behaviour corresponds to the critical case of the so-called coasting universe [11], [22]. In other words, classical strings provide a concrete matter realization of such cosmological model. Till now, no form of matter was known to describe coasting universes [11].

Finally, notice that strings continuously evolve from one type of behaviour to the other two. This is explicitly seen from the string solutions in refs. [2] - [⿴囗木 . For example the string described by $q_{-}(\sigma, \tau)$ for $\tau>0$ shows unstable behaviour for $\tau \rightarrow 0$, dual behaviour for $\tau \rightarrow \tau_{0}=1.246450 \ldots$ and stable behaviour for $\tau \rightarrow \infty$.

The equation of state for strings in four dimensional flat Minkowski spacetime is discussed in ref. [17]. One finds the values $4 / 3,2 / 3$ and 1 for $\gamma$ by choosing appropiate values of the average string velocity in chap. 7 of ref. [17].

\section{SELF-CONSISTENT STRING COSMOLOGY}

In the previous section we investigated the propagation of test strings in cosmological spacetimes. Let us now investigate how the Einstein equations in General Relativity and the effective equations of string theory (beta functions) can be verified self-consistently with our string solutions as sources.

We shall assume a gas of classical strings neglecting interactions as string splitting and coalescing. We will look for cosmological solutions described by metrics of the type (2.1). It 
is natural to assume that the background will have the same symmetry as the sources. That is, we assume that the string gas is homogeneous, described by a density energy $\rho=\rho\left(X^{0}\right)$ and a pressure $p=p\left(X^{0}\right)$. In the effective equations of string theory we consider a space independent dilaton field. Antisymmetric tensor fields wil be ignored.

\section{A. String Dominated Universes in General Relativity (no dilaton field)}

The Einstein equations for the geometry (2.1) take the form

$$
\begin{aligned}
\frac{1}{2}(D-1)(D-2) H^{2} & =\rho \quad, \\
(D-2) \dot{H}+p+\rho & =0
\end{aligned} .
$$

where $H \equiv \frac{d R}{d X^{0}} / R$. We know $p$ and $\rho$ as functions of $R$ in asymptotic cases. For large $R$, the unstable strings dominate [eq.(2.35)] and we have

$$
\rho=u R^{2-D} \quad, \quad p=-\frac{\rho}{D-1} \quad \text { for } R \rightarrow \infty
$$

For small $R$, the dual regime dominates with

$$
\rho=d R^{-D} \quad, \quad p=+\frac{\rho}{D-1} \quad \text { for } R \rightarrow 0
$$

We also know that stable solutions may be present with a contribution $\sim R^{1-D}$ to $\rho$ and with zero pressure. For intermediate values of $R$ the form of $\rho$ is clearly more complicated but a formula of the type

$$
\rho=\left(u R+\frac{d}{R}+s\right) \frac{1}{R^{D-1}}
$$

with $u, d$ and $s$ being positive constants, is qualitatively correct for all $R$ and becomes exact for $R \rightarrow 0$ and $R \rightarrow \infty$.

The pressure associated to the energy density (3.4) takes then the form

$$
p=\frac{1}{D-1}\left(\frac{d}{R}-u R\right) \frac{1}{R^{D-1}}
$$

Inserting eq.(3.5) into the Einstein-Friedmann equations [eq.(3.1)] we find

$$
\frac{1}{2}(D-1)(D-2)\left(\frac{d R}{d X^{0}}\right)^{2}=\left(u R+\frac{d}{R}+s\right) \frac{1}{R^{D-3}}
$$

We see that $R$ is a monotonic function of the cosmic time $X^{0}$. Eq.(3.6) yields

$$
X^{0}=\sqrt{\frac{(D-1)(D-2)}{2}} \int_{0}^{R} d R \frac{R^{D / 2-1}}{\sqrt{u R^{2}+d+s R}}
$$

where we set $R(0)=0$. 
It is easy to derive the behavior of $R$ for $X^{0} \rightarrow 0$ and for $X^{0} \rightarrow \infty$.

For $X^{0} \rightarrow 0, R \rightarrow 0$, the term $d / R$ dominates in eq.(3.6) and

$$
R\left(X^{0}\right) \stackrel{X^{0} \rightarrow 0}{\simeq} \frac{D}{2}\left[\frac{2 d}{(D-1)(D-2)}\right]^{\frac{1}{D}}\left(X^{0}\right)^{\frac{2}{D}}
$$

For $X^{0} \rightarrow \infty, R \rightarrow \infty$ and the term $u R$ dominates in eq.(3.6). Hence,

$$
R\left(X^{0}\right) \stackrel{X^{0}}{\simeq}\left[\frac{(D-2) u}{2(D-1)}\right]^{\frac{1}{D-2}}\left(X^{0}\right)^{\frac{2}{D-2}}
$$

For intermediate values of $X^{0}, R\left(X^{0}\right)$ is a continuous and monotonically increasing function of $X^{0}$.

In summary, the universe starts at $X^{0}=0$ with a singularity of the type dominated by radiation. (The string behaviour for $R \rightarrow 0$ is like usual radiation ). Then, the universe expands monotonically growing for large $X^{0}$ as $R \simeq\left[X^{0}\right]^{\frac{2}{D-2}}$. This is faster than (cold) matter dominated universes where $R \simeq\left[X^{0}\right]^{\frac{2}{D-1}}$. For example, for $D=4, R$ grows linearly with $X^{0}$ whereas for matter dominated universes $R \simeq\left[X^{0}\right]^{2 / 3}$.

As is clear already in eq.(3.6), for large $R$, unstable strings $(u R)$ dominate over the stable strings $(s)$. These stable strings behave as cold matter. It must be noticed that the qualitative form of the solution $R\left(X^{0}\right)$ does not depend on the particular positive values of $u, d$ and $s$.

We want to stress that we achieve a self-consistent solution of the Einstein equations with string sources since the behaviour of the string pressure and density given by eqs.(3.4)(3.5) precisely holds in universes with power like $R\left(X^{0}\right)$. Since we find positive exponents $K$ in the solution (3.9) both for small and large $R$, we can consistently ignore the solutions (2.32) which appear for $k<0$. It can be noticed that a linear growing behaviour but for all $D$ follows from the effective string equations (including the dilaton) without the string sources [15]. Notice that for string dominated universes, $R \simeq X^{0}$ appears only in $D=4$. On the other hand, the behaviour $R \simeq X^{0}$ in $D=4$ also appears, but for $X^{0} \rightarrow 0$ [23], in the context of the semiclassical Einstein equations using as source the four dimensional trace anomaly contribution to the energy-momentum tensor of quantum matter (point particle) fields.

\section{B. String Decay}

The unstable string solutions are called in this way since their energy and invariant length grow as $R$ for large $R$. However, it must be clear that as classical string solutions they never decay. The situation changes at the quantum level where strings may split and coalesce. Quantum string splitting calculations in Minkowski spacetime show that the splitting probability is proportional to the string length [19,20. This result holds at the critical dimension $(D=26)$ but it should be true more generally in absence of conformal anomalies (critical strings).

Since unstable strings are of invariant size proportional to $R$, this suggests that they will split with a growing probability as $R$ increases. Through splitting, two or more shorter 
strings will be produced which grow as $R$ if their size is $\sim R$. However, long strings often split into massless strings (radiation) and a long piece [20]; that is, into a 'dual' string with energy $\sim 1 / R$ and a long string with energy $\sim R$ for large $R$. This type of process is important because it reduces the number $(u)$ of unstable strings per comoving volume.

We can incorporate string decay in the string gas model of sec.3 A by considering $u$ and $d$ as functions of time $\left(X^{0}\right)$ in eqs. (3.4)-(3.5). From the above discussion the time derivative of $u$ must be proportional to the unstable string length $R$ and to $u$ itself:

$$
\frac{d u}{d X^{0}}=-\mathcal{C} R\left(X^{0}\right) u\left(X^{0}\right)
$$

where $\mathcal{C}$ is a positive constant of the order of the string coupling constant squared $(\sim$ $\left.e^{2 \phi_{0}}\right)$. The number $(d)$ of dual strings (massless quanta) per comoving volume increases as $R^{2}\left(-\frac{d u}{d X^{0}}\right)$ since an unstable string with energy $R$ yields $R^{2}$ quanta with energy $1 / R$ each. That is,

$$
\frac{d}{d X^{0}} d\left(X^{0}\right)=\mathcal{C} R\left(X^{0}\right)^{3} u\left(X^{0}\right)
$$

Eqs.(3.10)-(3.11) can be easily integrated:

$$
\begin{aligned}
& u\left(X^{0}\right)=u(0) \exp \left[-\mathcal{C} \int_{0}^{X^{0}} d t R(t)\right] \\
& d\left(X^{0}\right)=d(0)+\mathcal{C} \int_{0}^{X^{0}} d t R(t)^{3} u(t) .
\end{aligned}
$$

From eqs.(3.4),(3.5) and (3.12), we find for the total string density and pressure the following expressions,

$$
\begin{aligned}
& \rho=R\left(X^{0}\right)^{1-D}\left\{u(0) R\left(X^{0}\right) \exp \left[-\mathcal{C} \int_{0}^{X^{0}} d t R(t)\right]+\frac{1}{R\left(X^{0}\right)}\left[d(0)+\mathcal{C} \int_{0}^{X^{0}} d t R(t)^{3} u(t)\right]+s\right\} \\
& p=\frac{1}{D-1} R\left(X^{0}\right)^{1-D}\left\{\frac{1}{R\left(X^{0}\right)}\left[d(0)+\mathcal{C} \int_{0}^{X^{0}} d t R(t)^{3} u(t)\right]-u(0) R\left(X^{0}\right) \exp \left[-\mathcal{C} \int_{0}^{X^{0}} d t R(t)\right]\right\}
\end{aligned}
$$

A dramatic change happens for large expansion factors $R\left(X^{0}\right)$ since $u\left(X^{0}\right)$ decreases exponentially.

Notice that the pressure and energy density from eqs.(3.13) obey the continuity equation (2.8).

The Einstein-Friedmann equations [eqs.(3.6)] now become

$$
\begin{gathered}
\frac{1}{2}(D-1)(D-2)\left(\frac{d R}{d X^{0}}\right)^{2}=\left\{u(0) R\left(X^{0}\right) \exp \left[-\mathcal{C} \int_{0}^{X^{0}} d t R(t)\right]\right. \\
\left.+\frac{1}{R}\left[d(0)+\mathcal{C} \int_{0}^{X^{0}} d t R(t)^{3} u(t)\right]+s\right\} R\left(X^{0}\right)^{3-D}
\end{gathered}
$$

We see that $R\left(X^{0}\right)$ is always a monotonic function of $X^{0}$. It is easy to derive from eq.(3.14) the behaviour of $R\left(X^{0}\right)$ for small and for large $X^{0}$.

For $X^{0} \rightarrow \infty, R \rightarrow \infty$ and we find a matter dominated regime:

$$
R\left(X^{0}\right) \stackrel{X^{0}}{\simeq}\left[\frac{(D-1) s}{2(D-2)}\right]^{\frac{1}{D-1}} \quad\left(X^{0}\right)^{\frac{2}{D-1}}
$$

The unstable strings provide subdominant exponentially small corrections: 


$$
O\left(\left(X^{0}\right)^{\frac{2}{D-1}} \exp \left[-A\left(X^{0}\right)^{\frac{D+1}{D-1}}\right]\right) \quad, \quad \text { with } A=\mathcal{C}(s / 2)^{\frac{1}{D-1}}\left(\frac{D-1}{D-2}\right)^{\frac{D}{D-1}}
$$

Taking into account the string decay, the stable strings (which behave as cold matter) are those which remain for $R \rightarrow \infty$, and yield the realistic large $R$ behaviour (3.15). The dual strings for $R \rightarrow 0$ are not substantially affected by the string decay. For $X^{0} \rightarrow 0, R \rightarrow 0$ and we have again the radiation type behavior (3.8).

For very small splitting rate $\mathcal{C}$, unstable strings will decay very slowly and they can dominate $\rho$ for a while. Assuming this is the case, we can neglect the stable and dual pieces in eq.(3.13) and we find that $R\left(X^{0}\right)$ approaches a constant value $R_{1}$. Namely,

$$
R\left(X^{0}\right)=R_{1}-A e^{-\frac{1}{2} \mathcal{C} X^{0} R_{1}}, \text { for } X^{0} \geq 1 /\left[\mathcal{C} R_{1}\right], u(0) R_{1} \gg s
$$

where $A$ and $R_{1}$ are constant depending on the initial conditions. In other words, $R\left(X^{0}\right)$ reaches some plateau and then it grows again following the matter dominated behaviour (3.15).

\section{Thermodynamics of strings in cosmological spacetimes}

Let us consider a comoving volume $R^{D-1}$ filled by a gas of strings. The entropy change for this system is given by:

$$
T d S=d\left(\rho R^{D-1}\right)+p d\left(R^{D-1}\right)
$$

The continuity equation (2.8) and (3.18) implies that $d S / d t$ vanishes. That is, the entropy per comoving volume stays constant in time. Using now the thermodynamic relation [16]

$$
\frac{d p}{d T}=\frac{p+\rho}{T}
$$

it follows [17] that

$$
S=\frac{R^{D-1}}{T}(p+\rho)+\text { constant }
$$

Let us first ignore the possibility of string decay. Then, eq.(3.20) together with eqs.(3.4) and (3.5) yields the temperature as a function of the expansion factor $R$. That is,

$$
T=\frac{1}{S}\left\{s+\frac{1}{D-1}\left[\frac{D d}{R}+(D-2) u R\right]\right\}
$$

where $S$ stands for the (constant) value of the entropy.

Eq.(3.21) shows that for small $R, T$ scales as $1 / R$ whereas for large $R$ it scales as $R$. The small $R$ behaviour of $T$ is the usual exhibited by radiation. On the contrary, for large $\mathrm{R}$ the temperature grows proportional to R. This strange behaviour is actually absent when string splitting is considered.

Let us consider string decay. Inserting eq.(3.13) into eq.(3.20) yields: 
$T=\frac{1}{S}\left\{s+\frac{1}{D-1}\left[\frac{D}{R}\left(d(0)+\mathcal{C} \int_{0}^{X^{0}} d t R(t)^{3} u(t)\right)+(D-2) u(0) R \exp \left(-\mathcal{C} \int_{0}^{X^{0}} d t R(t)\right)\right]\right\}$

For small $R, T$ scales here as $1 / R$. For large $R$, stable strings $(s)$ dominates and one must take into account the temperature dependence of $s$ [17], in order to determine $T$ as a function of $R$.

\section{EFFECTIVE STRING EQUATIONS WITH THE STRING SOURCES INCLUDED}

Let us consider now the cosmological equations obtained from the low energy string effective action including the string matter as a classical source. In D spacetime dimensions, this action can be written as

$$
\begin{aligned}
S & =S_{1}+S_{2} \\
S_{1} & =\frac{1}{2} \int d^{D} x \sqrt{-G} e^{-\Phi}\left[R+G_{A B} \partial^{A} \Phi \partial^{B} \Phi+2 U(G, \Phi)-c\right] \\
S_{2} & =-\frac{1}{4 \pi \alpha^{\prime}} \sum_{\text {strings }} \int d \sigma d \tau G_{A B}(X) \partial_{\mu} X^{A} \partial^{\mu} X^{B}
\end{aligned}
$$

Here $A, B=0, \ldots, D-1$. This action is written in the so called 'Brans-Dicke frame' (BD) or 'string frame', in which matter couples to the metric tensor in the standard way. The BD frame metric coincides with the sigma model metric to which test strings are coupled.

Eq.4.1) includes the dilaton field $(\Phi)$ with a potential $U(G, \Phi)$ depending on the dilaton and graviton backgrounds; $c$ stands for the central charge deficit or cosmological constant term. The antisymmetric tensor field was not included, in fact it is irrelevant for the results obtained here. Extremizing the action (4.1) with respect to $G_{A B}$ and $\Phi$ yields the equations of motion

$$
\begin{gathered}
R_{A B}+\nabla_{A B} \Phi+2 \frac{\partial U}{\partial G_{A B}}-\frac{G_{A B}}{2}\left[R+2 \nabla^{2} \Phi-(\nabla \Phi)^{2}-c+2 U\right]=e^{\phi} T_{A B} \\
R+2 \nabla^{2} \Phi-(\nabla \Phi)^{2}-c+2 U-\frac{\partial U}{\partial \Phi}=0
\end{gathered}
$$

which can be more simply combined as

$$
\begin{aligned}
& R_{A B}+\nabla_{A B} \Phi+2 \frac{\partial U}{\partial G_{A B}}-G_{A B} \frac{\partial U}{\partial \Phi}=e^{\Phi} T_{A B} \\
& R+2 \nabla^{2} \Phi-(\nabla \Phi)^{2}-c+2 U-\frac{\partial U}{\partial \Phi}=0
\end{aligned}
$$

Here $T_{A B}$ stands for the energy momentum tensor of the strings as defined by eq.(2.6). It is also convenient to write these equations as

$$
R_{A B}-\frac{G_{A B}}{2} R=T_{A B}+\tau_{A B}
$$


where $\tau_{A B}$ is the dilaton energy momentum tensor :

$$
\tau_{A B}=-\nabla_{A B} \Phi+\frac{G_{A B}}{2}\left[2 \frac{\partial U}{\partial \Phi}-R\right]
$$

The Bianchi identity

$$
\nabla^{A}\left(R_{A B}-\frac{G_{A B}}{2} R\right)=0
$$

yields, as it must be, the conservation equation,

$$
\nabla^{A}\left(T_{A B}+\tau_{A B}\right)=0
$$

It must be noticed that eqs.(4.3) do not reduce to the Einstein equations of General Relativity

even when $\Phi=U=0$. Eqs. (4.3) yields in that case the Einstein equations plus the condition $R=0$.

\section{A. Effective String Equations in Cosmological Universes}

For the homogeneous isotropic spacetime geometries described by eq.(2.1) we have

$$
\begin{aligned}
R_{0}^{0} & =-(D-1)\left(\dot{H}+H^{2}\right) \\
R_{i}^{k} & =-\delta_{i}^{k}\left[\dot{H}+(D-1) H^{2}\right] \\
R & =-(D-1)\left(2 \dot{H}+D H^{2}\right) .
\end{aligned}
$$

where $H \equiv \frac{1}{R} \frac{d R}{d X^{0}}$.

The equations of motion (4.3) read

$$
\begin{array}{r}
\ddot{\Phi}-(D-1)\left(\dot{H}+H^{2}\right)-\frac{\partial U}{\partial \Phi}=e^{\Phi} \rho \\
\dot{H}+(D-1) H^{2}-H \dot{\Phi}+\frac{\partial U}{\partial \Phi}+\frac{R}{D-1} \frac{\partial U}{\partial R}=e^{\Phi} p \\
2 \ddot{\Phi}+2(D-1) H \dot{\Phi}-\dot{\Phi}^{2}-(D-1)\left(2 \dot{H}+D H^{2}\right)-2 \frac{\partial U}{\partial \Phi}-c+2 U=0
\end{array}
$$

where dot $\cdot$ stands for $\frac{d}{d X^{0}}$, and

$$
\rho=T_{0}^{0} \quad, \quad-\delta_{i}^{k} p=T_{i}^{k} .
$$

The conservation equation takes the form of eq.(2.8)

$$
\dot{\rho}+(D-1) H(p+\rho)=0
$$

By defining,

$$
\begin{aligned}
& \Psi \equiv \Phi-\log \sqrt{-G}=\Phi-(D-1) \log R \\
& \bar{\rho}=e^{\Phi} \rho \quad, \quad \bar{p}=e^{\Phi} p,
\end{aligned}
$$


equations (4.7) can be expressed in a more compact form as

$$
\begin{array}{r}
\ddot{\Psi}-(D-1) H^{2}-\left.\frac{\partial U}{\partial \Psi}\right|_{R}=\bar{\rho} \\
\dot{H}-H \dot{\Psi}+\left.\frac{R}{D-1} \frac{\partial U}{\partial R}\right|_{\Psi}=\bar{p} \\
\dot{\Psi}^{2}-(D-1) H^{2}-2 \bar{\rho}-2 U+c=0,
\end{array}
$$

The conservation equation reads

$$
\dot{\bar{\rho}}-\dot{\Psi} \bar{\rho}+(D-1) H \bar{p}=0
$$

As is known, under the duality transformation $R \longrightarrow R^{-1}$, the dilaton transforms as $\Phi \longrightarrow \Phi+(D-1) \log R$. The shifted dilaton $\Psi$ defined by eq.(4.10) is invariant under duality.

The transformation

$$
R^{\prime} \equiv R^{-1}
$$

implies

$$
\Psi^{\prime}=\Psi \quad, H^{\prime}=-H \quad, \bar{p}^{\prime}=-p \quad, \bar{\rho}^{\prime}=\bar{\rho}
$$

provided $u=d$, that is, a duality invariant string source. This is the duality invariance transformation of eqs.(4.11).

Solutions to the effective string equations have been extensively treated in the literature 21] and they are not our main purpose. For the sake of completeness, we briefly analyze the limiting behaviour of these equations for $R \rightarrow \infty$ and $R \rightarrow 0$.

It is difficult to make a complete analysis of the effective string equations (4.11) since the knowledge about the potential $U$ is rather incomplete. For weak coupling $\left(e^{\Phi}\right.$ small $)$ the supersymmetry breaking produces an effective potential that decreases very fast (as the exponential of an exponential of $\Phi)$ for $\Phi \rightarrow-\infty$.

Let us analyze the asymptotic behavior of eqs.(4.11) for $R \rightarrow \infty$ and $R \rightarrow 0$ assuming that the potential $U$ can be ignored. It is easy to see that a power behaviour Ansatz both for $R$ and for $e^{\Psi}$ as functions of $X^{0}$ is consistent with these equations. It turns out that the string sources do not contribute to the leading behaviour here, and we find for $R \rightarrow 0$

$$
\begin{aligned}
R_{\mp} & =C_{1}\left(X^{0}\right)^{ \pm 1 / \sqrt{D-1}} \rightarrow 0 \\
e^{\Psi_{\mp}} & =C_{2}\left(X^{0}\right)^{-1} \rightarrow\left\{\begin{array}{l}
\infty \\
0
\end{array}\right.
\end{aligned}
$$

Where $C_{1}$ and $C_{2}$ are constants. Here the branches $(-)$ and $(+)$ correspond to $X^{0} \rightarrow 0$ and to $X^{0} \rightarrow \infty$ respectively. In both regimes $R_{\mp} \rightarrow 0$ and $e^{\Phi_{\mp}} \rightarrow 0$.

The potential $U(\Phi)$ is hence negligible in these regimes. In terms of the conformal time $\eta$, the behaviours (4.15) result

$$
R_{\mp}=C_{1}^{\prime}(\eta)^{ \pm \frac{1}{\sqrt{D-1} \mp 1}} \rightarrow 0
$$




$$
e^{\Psi_{\mp}}=C_{2}^{\prime}(\eta)^{-\frac{\sqrt{D-1}}{\sqrt{D-1} \mp 1}} \rightarrow\left\{\begin{array}{l}
\infty \\
0
\end{array}\right.
$$

Where $C_{1}^{\prime}$ and $C_{2}^{\prime}$ are constants. The branch $(-)$ would describe an expanding noninflationary behaviour near the initial singularity $X^{0}=0$, while the branch $(+)$ describes a 'big crunch' situation and is rather unphysical.

Similarly, for $R \rightarrow \infty$ and $e^{\Phi} \rightarrow \infty$, we find

$$
\begin{aligned}
& R_{\mp}=D_{1}\left(X^{0}\right)^{\mp 1 / \sqrt{D-1}} \rightarrow \infty \\
& e^{\Psi_{\mp}}=D_{2}\left(X^{0}\right)^{-1} \rightarrow\left\{\begin{array}{l}
\infty \\
0
\end{array}\right.
\end{aligned}
$$

Where $D_{1}$ and $D_{2}$ are constants. Here again, the branches $(-)$ and $(+)$ correspond to $X^{0} \rightarrow 0$ and to $X^{0} \rightarrow \infty$ respectively, but now in both regimes $R_{\mp} \rightarrow \infty$ and $e^{\Phi_{\mp}} \rightarrow \infty$. (In this limit, one is not guaranteed that $U$ can be consistently neglected). In terms of the conformal time, eqs.(4.17) read

$$
\begin{array}{r}
R_{\mp}=D_{1}^{\prime}(\eta)^{\mp \frac{1}{\sqrt{D-1} \pm 1}} \rightarrow \infty \\
e^{\Psi_{\mp}}=D_{2}^{\prime}(\eta)^{-\frac{\sqrt{D-1}}{\sqrt{D-1} \pm 1}} \rightarrow\left\{\begin{array}{l}
\infty \\
0
\end{array}\right.
\end{array}
$$

The branch $(+)$ describes a noninflationary expanding behaviour for $X^{0} \rightarrow \infty$ faster than the standard matter dominated expansion, while the branch $(-)$ describes a super-inflationary behaviour $\eta^{-\alpha}$, since $0<\alpha<1$, for all $\mathrm{D}$.

The behaviours (4.15) for $R_{\mp} \rightarrow 0$ and (4.17) for $R_{\mp} \rightarrow \infty$ are related by duality $R \leftrightarrow 1 / R$.

\section{B. String driven inflation?}

Let us consider now the question of whether de Sitter spacetime may be a self-consistent solution of the effective string equations (4.7) with the string sources included. The strings in

cosmological universes like de Sitter spacetime have the equation of state (3.4)-(3.5). Since $e^{\Psi}=e^{\Phi} R^{1-D}:$

$$
\begin{aligned}
& \bar{\rho}=e^{\Psi}\left(u R+\frac{d}{R}+s\right) \\
& \bar{p}=\frac{e^{\Psi}}{D-1}\left(\frac{d}{R}-u R\right)
\end{aligned}
$$

In the absence of dilaton potential and cosmological constant term, the string sources do not generate de Sitter spacetime as discussed in sec. III.A. We see that for $U=c=0$, and $R=e^{H X^{0}}$, eqs.4.11) yields to a contradiction (unless $D=0$ ) for the value of $\Psi$, required to be $-H X^{0}+$ constant.

A self-consistent solution describing asymptotically de Sitter spacetime self-sustained by the string equation of state (4.19)-(4.20) is given by 


$$
\begin{aligned}
R & =e^{H X^{0}}, \quad H=\mathrm{constant}>0 \\
2 U-c & =D H^{2}=\mathrm{constant} \\
\Psi_{ \pm} & =\mp H X^{0} \pm i \pi+\log \frac{(D-1) H^{2}}{\rho_{ \pm}} \\
\rho_{+} & \equiv u \quad, \quad \rho_{-} \equiv d
\end{aligned}
$$

The branch $\Psi_{+}$describes the solution for $R \rightarrow \infty\left(X^{0} \rightarrow+\infty\right)$, while the branch $\Psi_{-}$ corresponds to $R \rightarrow 0\left(X^{0} \rightarrow-\infty\right)$. De Sitter spacetime with lorentzian signature selfsustained by the strings necessarily requires a constant imaginary piece $\pm i \pi$ in the dilaton field. This makes $e^{\Psi}<0$ telling us that the gravitational constant $G \sim e^{\Psi}<0$ here describes antigravity.

Is interesting to notice that in the euclidean signature case, i. e. $(+++\ldots++)$, the Ansatz $\dot{H}=0,2 U-c=$ constant, yields a constant curvature geometry with a real dilaton, but which is of Anti-de Sitter type. This solution is obtained from eqs.(4.20)-(4.21) through the transformation

$$
\hat{X}^{0}=i X^{0} \quad, \quad \hat{H}=-i H \quad, \quad X^{i}=X^{i} \quad, \quad \Psi=\Psi
$$

which maps the Lorentzian de Sitter metric into the positive definite one

$$
d \hat{s}^{2}=\left(d \hat{X}^{0}\right)^{2}+e^{\hat{H} \hat{X}^{0}}(d \vec{X})^{2} .
$$

The equations of motion (4.11) within the constant curvature Ansatz $(\dot{\hat{H}}=\ddot{\Psi}=0)$ are mapped onto the equations

$$
\begin{aligned}
(D-1) \hat{H}^{2}-\left.\frac{\partial U}{\partial \Psi}\right|_{R} & =\bar{\rho} \\
\hat{H} \frac{d \Psi}{d \hat{X}^{0}}+\left.\frac{R}{D-1} \frac{\partial U}{\partial R}\right|_{\Psi} & =\bar{p} \\
-\left(\frac{d \Psi}{d \hat{X}^{0}}\right)^{2}+(D-1) \hat{H}^{2}-2 \bar{\rho}-2 U+c & =0
\end{aligned}
$$

with the solution

$$
\begin{aligned}
R & =e^{\hat{H} \hat{X}^{0}}, \quad \hat{H}=\text { constant }>0, \\
c-2 U & =D \hat{H}^{2}=\text { constant } \\
\Psi_{ \pm} & =\mp \hat{H} \hat{X}^{0}+\log \frac{(D-1) \hat{H}^{2}}{\rho_{ \pm}} \\
\rho_{+} & \equiv u \quad, \quad \rho_{-} \equiv d
\end{aligned}
$$

Both solutions (4.25) and (4.21) are mapped one into another through the transformation (4.22).

It could be recalled that in the context of (point particle) field theory, de Sitter spacetime (as well as anti-de Sitter) emerges as an exact selfconsistent solution of the semiclassical Einstein equations with the back reaction included [24] - 25]. (Semiclassical in this context, means that matter fields including the graviton are quantized to the one-loop level and 
coupled to the (c-number) gravity background through the expectation value of the energymomentum tensor $T_{A}^{B}$. This expectation value is given by the trace anomaly: $\left\langle T_{A}^{A}\right\rangle=$ $\bar{\gamma} R^{2}$ ). On the other hand, the $\alpha^{\prime}$ expansion of the effective string action admits anti-de Sitter spacetime (but not de Sitter) as a solution when the quadratic curvature corrections (in terms of the Gauss-Bonnet term) to the Einstein action are included [26]. It appears that the corrections to the anti-de Sitter constant curvature are qualitatively similar in the both cases, with $\alpha^{\prime}$ playing the rôle of the trace anomaly parameter $\bar{\gamma}$ [25].

The fact that de Sitter inflation with true gravity $G \sim e^{\Psi}>0$ does not emerge as a solution of the effective string equations does not mean that string theory excludes inflation. What means is that the effective string equations are not enough to get inflation. The effective string action is a low energy field theory approximation to string theory containing only the massless string modes (massless background fields).

The vacuum energy scales to start inflation (physical or true vacuum) are typically of the order of the Planck mass [17] - [18] where the effective string action approximation breaks down. One must consider the massive string modes (which are absent from the effective string action) in order to properly get the cosmological condensate yielding de Sitter inflation. We do not have at present the solution of such problem. 


\section{REFERENCES}

[1] H. J. de Vega and N Sánchez, Phys. Rev. D47, 3394 (1993).

[2] H.J. de Vega, A.V. Mikhailov and N. Sánchez, Teor. Mat. Fiz. 94 (1993) 232.

[3] F. Combes, H.J. de Vega, A.V. Mikhailov and N. Sánchez, "Multi-String Solutions by Soliton Methods in de Sitter Spacetime", LPTHE 93-44/DEMIRM 93-58 preprint, to appear in Phys. Rev. D.

[4] H. J. de Vega, A L Larsen and N Sánchez, "Infinitely many strings in de Sitter spacetime: expanding and oscillating elliptic function solutions", LPTHE 93-56/DEMIRM 93-55 preprint.

[5] I. Krichever, "Two-dimensional algebraic-geometric operators with self-consistent potentials". Landau Institute preprint. To appear in Functional Analysis and Applications.

[6] H. J. de Vega and I. L. Egusquiza, Phys. Rev. D49, 763 (1994).

[7] A L Larsen and N Sánchez, 'Strings propagating in the $2+1$ black hole anti-de Sitter spacetime', DEMIRM 94013-preprint.

[8] N. Sánchez and G.Veneziano, Nucl. Phys. B333, 253 (1990).

[9] M. Gasperini, N. Sánchez and G.Veneziano, Int. J. Mod. Phys. A 6, 3853 (1991) and Nucl. Phys. B364, 365 (1991).

[10] H. J. de Vega and N. Sánchez, Int. J. Mod. Phys. A 7, 3043 (1992).

[11] G. F. R. Ellis, Banff Lectures 1990, in Gravitation, eds. R. Mann and P. Wesson, World Scientific 1991.

[12] See for a review, T.W.B. Kibble, Erice Lectures at the Chalonge School in Astrofundamental Physics, N. Sánchez editor, World Scientific, 1992.

[13] A. Vilenkin, Phys. Rev. D 24, 2082 (1981) and Phys. Rep. 121, 263 (1985). N. Turok and P. Bhattacharjee, Phys. Rev. D 29, 1557 (1984).

[14] J. D. Barrow, Nucl. Phys. B 310, 743 (1988).

[15] R. Myers, Phys. Lett. B199, 371 (1987). M. Mueller, Nucl. Phys. B337, 37 (1990). See for a review: A.A. Tseytlin in the Proceedings of the Erice School "String Quantum Gravity and Physics at the Planck Energy Scale", 21-28 June 1992, Edited by N. Sánchez, World Scientific, 1993.

[16] S. Weinberg, 'Gravitation and Cosmology', J. Wiley, 1972.

[17] E. W. Kolb and M. S. Turner, 'The Early Universe', Addison-Wesley, 1990.

[18] A. D. Linde, 'Particle Physics and Inflationary Cosmology', Harwood (1990).

[19] J. Polchinsky, Phys. Lett. B209, 252 (1988).

J. Day and J. Polchinsky, Phys. Lett. B220, 387 (1989).

[20] D. Mitchell, N. Turok, R. Wilkinson and P. Jetzer, Nucl. Phys. B 315, 1 (1989). D. Mitchell, B. Sundborg and N. Turok, Nucl. Phys. B 335, 621 (1990).

[21] See for example,

B. A. Campbell, A. Linde and K. A. Olive, Nucl. Phys. B 355, 146 (1991).

B. A. Campbell, N. Kaloper and K. A. Olive, Phys. Lett. B277, 265 (1992).

A.A. Tseytlin, Mod. Phys. Lett A 6, 1721 (1991).

A.A. Tseytlin and C. Vafa, Nucl. Phys. B 372, 443 (1992).

R. Brustein and P. J. Steinhardt, Phys. Lett. B302, 196 (1993).

M. Gasperini and G.Veneziano, Mod. Phys. Lett. A 8, 370 (1993),

Phys. Lett. B277, 256 (1992) and Astroparticle Physics 1, 317 (1993). 
E. Raiten, Nucl. Phys. B 416, 881 (1994).

R. Brustein and G.Veneziano, CERN TH 7179/94.

V. A. Kostelecký and M. J. Perry, Nucl. Phys. B 414, 174 (1994).

See in addition ref. [15].

[22] F. Müller-Holstein, Class. Quant. Grav. 3, 665 (1986).

K. G. Akdeniz et al. Mod. Phys. Lett. A 6, 1543 (1991) and

Phys. Lett. B 321, 329 (1994).

[23] M. V. Fischetti, J. B. Hartle and B. L. Hu, Phys. Rev. D 20, 1757 (1979).

We thank E. Calzetta for calling our attention to this paper.

[24] S. Wada and T. Azuma, Phys. Lett. B 132, 313 (1983).

V. Sahni and L. A. Kofman, Phys. Lett. A 117, 275 (1986).

[25] M. A. Castagnino, J. P. Paz and N. Sánchez, Phys. Lett. B 193, 13 (1987).

[26] D. G. Boulware and S. Deser, Phys. Rev. Lett. 55, 2656 (1985). 
Table I

STRING PROPERTIES FOR ARBITRARY $R\left(X^{0}\right)$

\begin{tabular}{|c|c|c|c|}
\hline & Energy & Pressure & $\begin{array}{l}\text { Equation of State: } \\
\quad p=(\gamma-1) \rho\end{array}$ \\
\hline \multicolumn{4}{|l|}{$\begin{array}{c}\mathrm{D}=1+1: \text { two families } \\
\text { of solutions }\end{array}$} \\
\hline $\begin{array}{l}\text { (i) } \eta \pm X=f_{ \pm}(\sigma \pm \tau) \\
\text { (ii) } \eta \pm X=\text { constant }\end{array}$ & $\begin{array}{l}E=u R \\
E=d / R\end{array}$ & $\begin{array}{l}P=-E \\
P=+E\end{array}$ & $\begin{array}{l}\gamma=0 \\
\gamma=2\end{array}$ \\
\hline $\begin{array}{l}D=2+1 \text { : Ring Solutions, } \\
\text { three asymptotic } \\
\text { behaviours }(\mathrm{u}, \mathrm{d}, \mathrm{s})\end{array}$ & $E=\frac{1}{\alpha^{\prime}} \dot{X}^{0}(\tau)$ & $P=\frac{R(\tau)^{2}}{2 \alpha^{\prime}\left|\dot{X}^{0}(\tau)\right|}\left[\dot{f}^{2}-f^{2}\right]$ & \\
\hline $\begin{array}{l}\text { (i) unstable for } R \rightarrow \infty \\
\text { (ii) dual to (i) for } R \rightarrow 0 \\
\text { (iii) stable for } R \rightarrow \infty\end{array}$ & $\begin{array}{l}E_{u} \stackrel{R \rightarrow \infty}{=} u R \rightarrow \infty \\
E_{d} \stackrel{R \rightarrow 0}{=} d / R \rightarrow \infty \\
E_{s}=\text { constant }\end{array}$ & $\begin{array}{l}P_{u}=-E / 2 \rightarrow-\infty \\
P_{d}=+E / 2 \rightarrow \infty \\
P_{s}=0\end{array}$ & $\begin{array}{l}\gamma_{u}=1 / 2 \\
\gamma_{d}=3 / 2 \\
\gamma_{s}=1\end{array}$ \\
\hline \multicolumn{4}{|l|}{$\begin{array}{l}\text { D-Dimensional spacetimes: } \\
\text { general asymptotic behaviour }\end{array}$} \\
\hline $\begin{array}{l}\text { (i) unstable for } R \rightarrow \infty \\
\text { (ii) dual to (i) for } R \rightarrow 0 \\
\text { (iii) stable for } R \rightarrow \infty\end{array}$ & $\begin{array}{l}E_{u} \stackrel{R \rightarrow \infty}{=} \text { u } R \rightarrow \infty \\
E_{d} \stackrel{R \rightarrow 0}{=} d / R \rightarrow \infty \\
E_{s}=\text { constant }\end{array}$ & $\begin{array}{l}P_{u}=-\frac{E}{D-1} \rightarrow-\infty \\
P_{d}=+\frac{E}{D-1} \rightarrow \infty \\
P_{s}=0\end{array}$ & $\begin{array}{l}\gamma_{u}=(D-2) /(D-1) \\
\gamma_{d}=D /(D-1) \\
\gamma_{s}=1\end{array}$ \\
\hline
\end{tabular}


Table II

STRING ENERGY DENSITY AND PRESSURE FOR ARBITRARY $R\left(X^{0}\right)$

\begin{tabular}{|l|l|l|}
\hline & Energy density: $\rho \equiv E / R^{D-1}$ & Pressure \\
\hline $\begin{array}{l}\text { Qualitatively correct } \\
\text { formulas for all } \mathrm{R} \text { and } \mathrm{D}\end{array}$ & $\rho=\left(u R+\frac{d}{R}+s\right) \frac{1}{R^{D-1}}$ & $p=\frac{1}{D-1}\left(\frac{d}{R}-u R\right) \frac{1}{R^{D-1}}$ \\
\hline
\end{tabular}

Table III

\section{STRING COSMOLOGY IN GENERAL RELATIVITY}

\begin{tabular}{|l|c|c|}
\hline $\begin{array}{l}\text { Einstein equations } \\
(\text { no dilaton field })\end{array}$ & $\begin{array}{c}\text { Expansion factor } \\
R\left(X^{0}\right)\end{array}$ & $\begin{array}{c}\text { Temperature } \\
T(R)\end{array}$ \\
\hline$X^{0} \rightarrow 0$ & $\frac{D}{2}\left[\frac{2 d}{(D-1)(D-2)}\right]^{\frac{1}{D}}\left(X^{0}\right)^{\frac{2}{D}}$ & $\frac{d D}{S(D-1)} 1 / R$ \\
\hline $\begin{array}{l}X^{0} \rightarrow \infty \\
(\text { without string decay })\end{array}$ & {$\left[\frac{(D-2) u}{2(D-1)}\right]^{\frac{1}{D-2}}\left(X^{0}\right)^{\frac{2}{D-2}}$} & $\frac{(D-2) u}{(D-1) S} R$ \\
\hline $\begin{array}{l}X^{0} \rightarrow \infty \\
(\text { with string splitting })\end{array}$ & {$\left[\frac{(D-1) s}{2(D-2)}\right]^{\frac{1}{D-1}}\left(X^{0}\right)^{\frac{2}{D-1}}$} & $\begin{array}{l}\text { usual matter } \\
\text { dominated behaviour }\end{array}$ \\
\hline
\end{tabular}


Table IV

EFFECTIVE STRING EQUATIONS SOLUTIONS IN COSMOLOGY

\begin{tabular}{|c|c|c|}
\hline $\begin{array}{c}\text { Effective String } \\
\text { equations }\end{array}$ & $\begin{array}{c}R\left(X^{0}\right) \rightarrow 0 \\
\text { behaviour }\end{array}$ & $\begin{array}{c}R\left(X^{0}\right) \rightarrow \infty \\
\text { behaviour }\end{array}$ \\
\hline$X^{0} \rightarrow 0$ & $\sim\left(X^{0}\right)^{+1 / \sqrt{D-1}}$ & $\sim\left(X^{0}\right)^{-1 / \sqrt{D-1}}$ \\
\hline$X^{0} \rightarrow \infty$ & $\sim\left(X^{0}\right)^{-1 / \sqrt{D-1}}$ & $\sim\left(X^{0}\right)^{+1 / \sqrt{D-1}}$ \\
\hline
\end{tabular}

\section{Table Captions}

Table I. String energy and pressure as obtained from exact string solutions for various expansion factors $R\left(X^{0}\right)$.

Table II. The string energy density and pressure for a gas of strings can be summarized by the above formulas which become exact for $R \rightarrow 0$ and for $R \rightarrow \infty$.

Table III. The self-consistent cosmological solution of the Einstein equations in General Relativity with the string gas as source.

Table IV. Asymptotic solution of the string effective equations (including the dilaton). 